\title{
Evaluation of efficacy and nephrotoxicity during vancomycin therapy: A retrospective study in China
}

\author{
LIPING WANG $^{1 *}$, QING YUAN ${ }^{2 *}$, MIN TAN $^{1}$, SHUANSHUAN XIE $^{1}$, \\ JUFANG WU ${ }^{3}$, XIAOLIAN SONG ${ }^{1}$ and CHANGHUI WANG ${ }^{1}$
}

\begin{abstract}
Departments of ${ }^{1}$ Respiratory Medicine and ${ }^{2}$ Emergency Medicine, Shanghai 10th People's Hospital, Tongji University, Shanghai 200072; ${ }^{3}$ Institute of Antibiotics, Huashan Hospital, Fudan University, Shanghai 200040, P.R. China
\end{abstract}

Received May 23, 2018; Accepted November 23, 2018

DOI: $10.3892 /$ etm.2019.7188

\begin{abstract}
The aim of the present study was to investigate the predictive value of vancomycin serum concentrations regarding its efficacy and nephrotoxicity in a Chinese population and to determine a relatively safe optimal target concentration during vancomycin therapy. A total of 65 patients that received vancomycin between March 2013 and March 2018 at Shanghai 10th People's Hospital (Shanghai, China) were enrolled and their vancomycin trough and peak concentrations were monitored. Factor analysis was performed in order to exclude interaction between variables. Univariate and multivariate analyses were used to identify predictors of drug efficacy and nephrotoxicity. Receiver operating characteristic curve analysis was performed to determine the thresholds of the vancomycin trough and peak concentrations for optimal efficacy and acceptable nephrotoxicity, respectively. Among the 65 cases, treatment was deemed to be effective for 43 patients and ineffective for 22 patients. Furthermore, 20 patients fulfilled the criteria for nephrotoxicity. A total of 15 continuous variables loaded the first five factors by factor analysis (which converts large numbers of highly inter-correlated variables into a small number of comprehensive indicators that reflect a dimensionality reduction) and the factors were as follows: Inflammation, renal function, liver function, vancomycin trough and peak concentrations, and nutritional status. Univariate and multivariate analyses identified the trough concentration and peak concentration as independent variables associated with efficacy and nephrotoxicity of vancomycin, and the nutritional status was a risk factor associated with efficacy. Regarding efficacy, the critical values for the trough concentration and
\end{abstract}

Correspondence to: Dr Xiaolian Song, Department of Respiratory Medicine, Shanghai 10th People's Hospital, Tongji University, 301 Yangchang Middle Road, Shanghai 200072, P.R. China

E-mail: alian818@hotmail.com

*Contributed equally

Key words: vancomycin, therapeutic drug monitoring, efficacy, nephrotoxicity peak concentration were determined to be $9.02 \mathrm{mg} / \mathrm{l}(95.3 \%$ sensitivity and $68.2 \%$ specificity) and $23.62 \mathrm{mg} / \mathrm{l}(83.7 \%$ sensitivity and $59.1 \%$ specificity), respectively. The thresholds of vancomycin trough and peak concentrations for the development of nephrotoxicity were $16.08 \mathrm{mg} / \mathrm{l}(80.0 \%$ sensitivity and $84.4 \%$ specificity) and $30.42 \mathrm{mg} / \mathrm{l}$ (75.0\% sensitivity and $73.3 \%$ specificity), respectively. In conclusion, during vancomycin therapy, the trough and peak concentrations are associated with efficacy and nephrotoxicity. Furthermore, a trough concentration between 9.02 and $16.08 \mathrm{mg} / \mathrm{l}$ and a peak concentration of 23.62-30.42 mg/l were determined to be relatively safe (the clinical trial registry no. ChiCTR-OPC-16007920).

\section{Introduction}

Staphylococcus aureus, the most common type of Gram-positive coccus, is an important pathogenic bacterium. It is widely distributed in the environment and has strong pathogenicity; it is able to cause a number of common or complex infectious diseases, including pneumonia, arthritis, urinary tract infections, osteomyelitis and meningitis. Methicillin-resistant $S$. aureus (MRSA) accounts for $>55 \%$ of all $S$. aureus infections in communal and health care-associated settings (1). With the prevalence of MRSA infections increasing, its morbidity, mortality and cost of medical care are increased (2).

Vancomycin, a glycopeptide antibiotic, is the first-line agent in the treatment of $S$. aureus strains that produce penicillinase, particularly for patients infected with MRSA (3-5). However, vancomycin is almost exclusively eliminated by the kidneys; therefore, a potentially serious adverse effect of vancomycin is nephrotoxicity $(6,7)$. Optimization of vancomycin therapy with therapeutic drug monitoring (TDM) may improve the treatment efficacy, and avoid nephrotoxicity and drug resistance $(8,9)$. It has been determined that the superior effect of vancomycin is highly correlated with the area under the concentration-time curve (AUC) and the minimum inhibitory concentration (MIC). However, it is difficult to obtain the AUC in the clinical setting. Thus, vancomycin serum trough concentrations may be used as a substitute for AUCs (10). A consensus review published by the Infectious Diseases Society of America (IDSA) provides recommendations that serum vancomycin trough concentrations should always be 
maintained at $>10 \mathrm{mg} / \mathrm{l}$ in order to avoid the development of vancomycin resistance in adult patients, and a vancomycin serum trough concentration of $15-20 \mathrm{mg} / \mathrm{l}$ is recommended for complicated infections (3). However, certain studies have indicated that higher vancomycin trough concentrations $(\geq 15 \mathrm{mg} / \mathrm{l}$ ) are associated with higher rates of nephrotoxicity. Furthermore, the results of studies on vancomycin TDM in China indicate that the dosage of vancomycin is generally low $(11,12)$. Therefore, there is controversy regarding the optimal target concentration of vancomycin.

The present study performed a retrospective analysis in order to investigate the predictive value of vancomycin serum concentrations regarding the efficacy and nephrotoxicity in patients in China and to determine a relatively safe optimal target concentration during vancomycin therapy.

\section{Patients and methods}

Study design and patients. Hospitalized patients who received a course of vancomycin therapy between March 2013 and March 2018 at the Department of Respiratory Medicine of Shanghai 10th People's Hospital Affiliated to the Tongji University (Shanghai, China) were retrospectively reviewed. The inclusion criteria were as follows: i) Vancomycin therapy for at least 3 days; ii) requirement of TDM of vancomycin to assess efficacy and toxicity and iii) written informed consent. The exclusion criteria were as follows: i) Treatment with vancomycin within $72 \mathrm{~h}$ prior to the monitoring phase; ii) pregnant or breastfeeding women; iii) no availability of the laboratory data; iv) patients with diseases affecting the metabolism of vancomycin.

Data collection. The investigators observed the patients daily during vancomycin therapy until it was discontinued or the patient was discharged from the hospital, depending on which happened first. During the observation period, the following demographic information was collected: Gender, age, weight, height, diagnosis, the site of the Gram-positive cocci culture, length of hospitalization and whether the patient had undergone surgery, been implanted with medical devices or admitted to an intensive care unit (ICU). The vancomycin trough and peak concentrations were recorded. Trough concentrations were obtained just prior to the subsequent dose under steady-state conditions (approximately after the fourth dose). Peak concentration monitoring was performed $0.5-1 \mathrm{~h}$ after the end of the fifth dose. Laboratory values, medical history and comorbidities, concomitant medications (carbapenems, cephalosporins, aminoglycosides and quinolones), microbiologic data and details regarding vancomycin treatment (date, time, dosing regimen, initial dosing frequency and duration) were noted on a daily basis.

Definitions. The definition of comprehensive efficacy included the results of clinical efficacy evaluation and bacteriological efficacy evaluation as follows: The clinical symptoms and signs, as well as the radiologic and laboratory tests (including bacteriology) returned to normal or pre-infection status; and vancomycin was not required within 7 days after discontinuation of the drug. In the primary analysis, three definitions of nephrotoxicity were used: i) An increase in serum creatinine (SCr) to $\geq 0.5 \mathrm{mg} / \mathrm{dl}(44.2 \mathrm{mmol} / \mathrm{l})$; and ii) a $50 \%$ increase in
$\mathrm{SCr}$; or iii) a $25 \%$ reduction in estimated creatinine clearance $(\mathrm{CrCl})$ from the baseline level for $\geq 2$ days. Collection of $\mathrm{SCr}$ values commenced prior to the start of vancomycin treatment and continued until $72 \mathrm{~h}$ after the treatment was completed. The $\mathrm{CrCl}$ value was estimated using the Cockcroft-Gault formula (13).

Statistical analysis. Data analysis was performed using SPSS Statistics software, version 20.0 (IBM Corp., Armonk, NY, USA). For the univariate analysis, Pearson's Chi-square test or Fisher's exact test were used to compare categorical variables, and Student's t-test or the Mann-Whitney U-test were used to compare continuous variables. Logistic regression analyses were used to identify predictors of efficacy and nephrotoxicity. Receiver operating characteristic (ROC) curve analysis was used to determine the thresholds of the vancomycin trough and peak concentrations for efficacy and nephrotoxicity, respectively. Values are expressed as the mean \pm standard deviation. For all analyses, $\mathrm{P} \leq 0.05$ was considered to indicate a statistically significant difference and all tests were two-tailed.

Selection of variables for analysis. In order to select variables for the regression model with the intent of minimizing multicollinearity, factor analysis was further performed on all continuous variables to reduce interaction between variables with orthogonal varimax rotation (14). Scree plots were used to describe the importance of the factors, and the number of components retained in the rotated structure was based on Jolliffe's criterion that eigenvalues should be $>0.70$ (15). The results are presented as rotated factor loadings, and the variables were sorted by factor according to the highest loading.

\section{Results}

Patient characteristics. The patients treated at Shanghai 10th People's Hospital (Shanghai, China) between March 2013 and March 2018 who met the inclusion criteria but not the exclusion criteria $(n=65)$ were retrospectively enrolled in the present study. Among them, 38 were male and 27 were female, and the mean age was $61.9 \pm 20.1$ years. Of these patients, 40 were admitted to the ICU. The primary site of infection was the lungs $(80.0 \%)$ and the bloodstream $(15.0 \%)$, while others accounted for $7.1 \%$. Cardiovascular diseases $(53.8 \%)$ and diabetes $(24.6 \%)$ accounted for a large proportion of the underlying diseases. The mean vancomycin serum trough concentration and peak concentration were $13.7 \pm 9.1$ and $28.2 \pm 8.7 \mathrm{mg} / \mathrm{l}$, respectively. The mean total dosage of vancomycin was $19.5 \pm 12.2 \mathrm{~g}$. The demographics and clinicopathological characteristics of the patients are presented in Table I.

Factor analysis. The first five comprehensive indicators representing 15 continuous variables were loaded by factor analysis with orthogonal varimax rotation, accounting for $75.7 \%$ of the total information (Table II). The importance of the factors was determined using scree plots and sorting of variables according to the largest absolute loading (Fig. 1). The rotated component matrix is presented in Table III and the results were as follows: Factor 1 was associated with inflammation (the percentage of neutrophils and lymphocytes); factor 2 was highly associated with the renal function [baseline blood urea neutrogen (BUN), 
Table I. Demographics and clinicopathological characteristics of patients in the effective and ineffective treatment groups.

\begin{tabular}{|c|c|c|c|c|}
\hline Characteristic/parameter & Total $(n=65)$ & Effective group $(n=43)$ & Ineffective group $(n=22)$ & P-value \\
\hline Males & $38(58.5)$ & $26(60.5)$ & $12(54.5)$ & 0.647 \\
\hline Age (years) & $61.9 \pm 20.1$ & $60.7 \pm 20.1$ & $64.2 \pm 20.2$ & 0.509 \\
\hline BMI $\left(\mathrm{kg} / \mathrm{m}^{2}\right)$ & $22.5 \pm 4.0$ & $23.4 \pm 3.6$ & $20.7 \pm 4.2$ & 0.010 \\
\hline \multicolumn{5}{|l|}{ Baseline laboratory parameters } \\
\hline $\mathrm{BUN}(\mathrm{mmol} / \mathrm{l})$ & $8.0 \pm 5.1$ & $6.8 \pm 3.5$ & $10.3 \pm 6.8$ & 0.009 \\
\hline $\operatorname{Scr}(\mu \mathrm{mol} / \mathrm{l})$ & $75.2 \pm 41.6$ & $76.0 \pm 40.2$ & $74.0 \pm 45.2$ & 0.854 \\
\hline $\mathrm{CrCl}(\mathrm{ml} / \mathrm{min})$ & $96.2 \pm 50.8$ & $95.6 \pm 41.9$ & $97.4 \pm 66.1$ & 0.892 \\
\hline $\operatorname{ALT}(\mathrm{U} / \mathrm{l})$ & $34.0 \pm 38.4$ & $27.8 \pm 15.0$ & $46.2 \pm 61.7$ & 0.066 \\
\hline AST (U/l) & $40.3 \pm 35.5$ & $33.6 \pm 26.0$ & $53.7 \pm 47.0$ & 0.030 \\
\hline WBC $\left(10^{9} / 1\right)$ & $10.8 \pm 5.6$ & $10.7 \pm 5.7$ & $11.0 \pm 5.7$ & 0.808 \\
\hline Neutrophils (\% in WBCs) & $80.0 \pm 11.1$ & $79.7 \pm 12.1$ & $80.7 \pm 9.0$ & 0.854 \\
\hline Lymphocytes (\% in WBCs) & $15.8 \pm 9.4$ & $16.0 \pm 10.0$ & $15.5 \pm 8.2$ & 0.733 \\
\hline Albumin $(\mathrm{g} / \mathrm{l})$ & $30.2 \pm 6.3$ & $31.7 \pm 6.0$ & $27.4 \pm 6.2$ & 0.009 \\
\hline Baseline body temperature $\left({ }^{\circ} \mathrm{C}\right)$ & $38.0 \pm 1.0$ & $38.0 \pm 1.0$ & $38.0 \pm 0.9$ & 0.856 \\
\hline ICU patients & $40(61.5)$ & $23(53.5)$ & $17(77.3)$ & 0.062 \\
\hline Primary site of infection & & & & 0.732 \\
\hline Respiratory tract & $16(80.0)$ & $34(75.6)$ & & \\
\hline Bloodstream & $3(15.0)$ & $6(13.3)$ & & \\
\hline Others & $1(5)$ & $5(11.1)$ & & \\
\hline \multicolumn{5}{|l|}{ Underlying disease } \\
\hline Cardiovascular & $35(53.8)$ & $26(60.5)$ & $9(40.9)$ & 0.109 \\
\hline Diabetes & $16(24.6)$ & $13(30.2)$ & $3(13.6)$ & 0.224 \\
\hline \multicolumn{5}{|l|}{ Vancomycin concentration (mg/l) } \\
\hline Trough & $13.9 \pm 7.1$ & $16.3 \pm 6.3$ & $9.0 \pm 6.2$ & $<0.001$ \\
\hline Trough $\leq 15$ & $32(49.2)$ & $14(32.6)$ & $18(81.8)$ & $<0.001$ \\
\hline Trough $>15$ & $33(50.8)$ & $29(67.4)$ & $4(18.2)$ & \\
\hline Peak & $28.2 \pm 8.7$ & $30.4 \pm 7.8$ & $23.8 \pm 8.8$ & 0.003 \\
\hline Total vancomycin dose (g) & $19.7 \pm 12.1$ & $20.8 \pm 12.8$ & $17.6 \pm 10.3$ & 0.313 \\
\hline
\end{tabular}

Values are expressed as $\mathrm{n}(\%)$ or the mean \pm standard deviation. BMI, body mass index (normal range, 18.5-24.9 kg/m²); BUN, blood urea nitrogen (normal range, 3.2-7.1 mmol/l); Scr, serum creatinine (normal range, 44-133 $\mu \mathrm{mol} / \mathrm{l}$ ); $\mathrm{CrCl}$, creatinine clearance (normal range, 80-120 ml/min); ALT, alanine aminotransferase (normal range, 5-40 U/l); AST, aspartate transaminase (normal range, 8-40 U/l); WBC, white blood cell (normal range, 4-10x10\%/l); ICU, intensive care unit. Neutrophil normal range, 50-70\%, lymphocytes normal range, 20-40\% and albumin normal range, $35-50 \mathrm{~g} / \mathrm{l}$.

Table II. Demographics and characteristics loaded over five factors explaining $75.7 \%$ of the information.

\begin{tabular}{lll}
\hline Factor & $\begin{array}{c}\text { Variance } \\
\text { explained }(\%)^{\mathrm{a}}\end{array}$ & \multicolumn{1}{c}{ Factors } \\
\hline 1 & 24.9 & Baseline neutrophils, lymphocytes \\
2 & 16.8 & $\begin{array}{l}\text { Baseline BUN, Scr, CrCl } \\
3\end{array}$ \\
4 & 14.4 & Baseline ALT, AST \\
& 10.4 & $\begin{array}{l}\text { Vancomycin trough concentration, } \\
\text { peak concentration } \\
5\end{array}$ \\
& 9.2 & BMI, baseline albumin \\
\hline
\end{tabular}

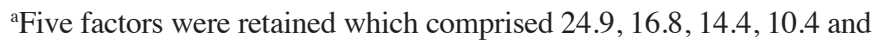
$9.2 \%$ of the total variance, respectively. BMI, body mass index; BUN, blood urea nitrogen; Scr, serum creatinine; $\mathrm{CrCl}$, creatinine clearance; ALT, alanine aminotransferase; AST, aspartate transaminase.
$\mathrm{SCr}$ and $\mathrm{CrCl}$ ]; factor 3 was mainly associated with the liver function [baseline alanine aminotransferase and aspartate transaminase (AST)]; factor 4 was mainly determined by the vancomycin trough and peak concentrations; and factor 5 was mostly associated with the nutritional status [body mass index (BMI) and baseline albumin].

Parameters influencing the efficacy of vancomycin. Of the 65 eligible patients, vancomycin treatment was rated to be effective in 43 patients and ineffective in 22 patients. Overall, the effective group and the ineffective group were similar regarding the majority or clinicopathological and demographic parameters, but the BMI $(\mathrm{P}=0.010)$, baseline BUN $(\mathrm{P}=0.009)$, baseline AST $(\mathrm{P}=0.030)$, baseline albumin $(\mathrm{P}=0.009)$, vancomycin trough concentration $(\mathrm{P}<0.001)$ and vancomycin peak concentration $(\mathrm{P}=0.003)$ were significantly different between the effective group and the ineffective group. Stratification of the patients 
Table III. Factor loadings in the total patients.

\begin{tabular}{|c|c|c|c|c|c|}
\hline Variable & Factor 1 & Factor 2 & Factor 3 & Factor 4 & Factor 5 \\
\hline Age & -0.546 & 0.058 & -0.512 & 0.262 & -0.289 \\
\hline Vancomycin trough concentration & -0.041 & 0.093 & -0.051 & 0.893 & 0.121 \\
\hline Vancomycin peak concentration & 0.065 & -0.005 & -0.200 & 0.869 & -0.013 \\
\hline Baseline BUN & -0.054 & 0.806 & -0.136 & -0.15 & -0.079 \\
\hline Baseline Scr & 0.237 & 0.895 & -0.023 & 0.156 & 0.183 \\
\hline Baseline $\mathrm{CrCl}$ & 0.319 & -0.758 & 0.269 & -0.261 & 0.141 \\
\hline BMI & 0.245 & -0.214 & 0.068 & 0.193 & 0.832 \\
\hline Total vancomycin dose & 0.273 & -0.559 & -0.365 & 0.115 & 0.466 \\
\hline Baseline WBC & -0.162 & 0.285 & -0.142 & -0.337 & 0.557 \\
\hline Baseline neutrophils & -0.976 & 0.036 & -0.042 & -0.071 & -0.048 \\
\hline Baseline lymphocytes & 0.956 & -0.047 & 0.015 & 0.028 & 0.085 \\
\hline Baseline ALT & 0.214 & -0.025 & 0.830 & -0.052 & -0.017 \\
\hline Baseline AST & 0.045 & -0.058 & 0.667 & -0.100 & 0.172 \\
\hline Baseline albumin & 0.109 & -0.001 & 0.519 & 0.120 & 0.700 \\
\hline Baseline body temperature & -0.263 & -0.220 & 0.593 & -0.094 & -0.159 \\
\hline
\end{tabular}

BMI, body mass index; BUN, blood urea nitrogen; Scr, serum creatinine; CrCl, creatinine clearance; ALT, alanine aminotransferase; AST, aspartate transaminase; WBC, white blood cell.

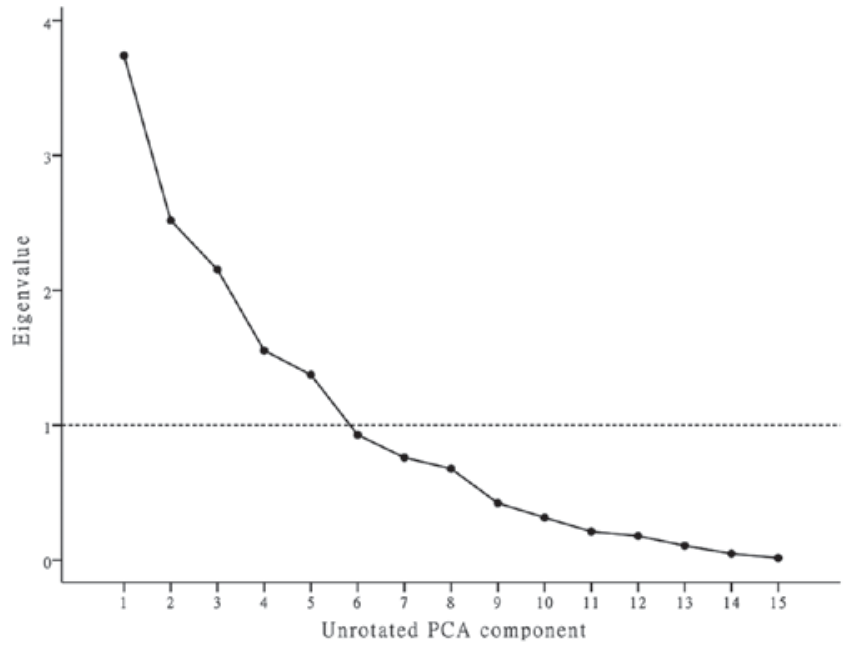

Figure 1. Scree plot of the full data set. Factors with eigenvalues $>1$ in the scree plot were retained. PCA, principal component analysis.

according to high and low trough concentration indicated that the frequency of ineffective treatment in the low (trough concentration, $\leq 15 \mathrm{mg} / \mathrm{l}$ ) group (81.8\%) was markedly higher than that in the high (trough concentration, $>15 \mathrm{mg} / \mathrm{l}$ ) group (18.2\%). Analysis with the Chi-squared test indicated a high association between efficacy and a trough concentration of $>15 \mathrm{mg} / \mathrm{l}(\mathrm{P}<0.001$; Table I).

Logistic regression analysis for efficacy. Logistic regression analysis was performed using the dimensional data reduced by factor analysis. The results confirmed that factor 4 [odds ratio $(\mathrm{OR})=5.480 ; 95 \%$ confidence interval $(\mathrm{CI}): 1.734-17.325$; $\mathrm{P}=0.004]$ and factor $5(\mathrm{OR}=3.164 ; 95 \% \mathrm{CI}: 1.002-9.987$; $\mathrm{P}=0.037)$ were independent influencing factors regarding efficacy. The other factors were not significantly associated with
Table IV. Logistic regression analyses of independent influencing factors for efficacy in all subjects $(n=65)$.

\begin{tabular}{llll}
\hline Factor & OR for efficacy & \multicolumn{1}{c}{$95 \% \mathrm{CI}$} & P-value \\
\hline 1 & 1.703 & $0.566-5.127$ & 0.344 \\
2 & 0.566 & $0.253-1.266$ & 0.166 \\
3 & 0.953 & $0.509-1.783$ & 0.879 \\
4 & 5.480 & $1.734-17.325$ & 0.004 \\
5 & 3.164 & $1.002-9.987$ & 0.037 \\
\hline
\end{tabular}

OR, odds ratio; $\mathrm{CI}$, confidence interval.

the efficacy and were therefore not included in the final model. Hence, the BMI, baseline albumin, and vancomycin trough and peak concentrations of the patients were associated with the efficacy of vancomycin (Table IV).

Parameters influencing the nephrotoxicity of vancomycin. Among the 65 patients, 20 met the criteria for nephrotoxicity. The baseline body temperature $(\mathrm{P}=0.014)$, total vancomycin dose $(\mathrm{P}=0.041)$, trough concentration $(\mathrm{P}<0.001)$ and peak concentration $(\mathrm{P}=0.020)$ exhibited significant differences between the groups of patients with and without nephrotoxicity. A significant difference in nephrotoxicity was also noted between the low and high trough concentration groups $(\mathrm{P}<0.001)$. The incidence of nephrotoxicity was only $15.0 \%$ in the low trough concentration group but $85.0 \%$ in the high trough concentration group (Table V).

Logistic regression analysis for nephrotoxicity. Logistic regression analysis for nephrotoxicity identified a significant 
Table V. Comparison of characteristics between patients with and without nephrotoxicity (total $n=65$ ).

\begin{tabular}{|c|c|c|c|}
\hline Characteristic & Nephrotoxicity group $(n=20)$ & Non-nephrotoxicity group $(\mathrm{n}=45)$ & P-value \\
\hline Males & $14(70.0)$ & $24(53.3)$ & 0.208 \\
\hline Age (years) & $66.7 \pm 18.1$ & $59.8 \pm 20.7$ & 0.207 \\
\hline BMI $\left(\mathrm{kg} / \mathrm{m}^{2}\right)$ & $23.0 \pm 3.96$ & $22.3 \pm 4.0$ & 0.497 \\
\hline \multicolumn{4}{|l|}{ Baseline laboratory parameters } \\
\hline BUN (mmol/l) & $7.5 \pm 3.5$ & $8.2 \pm 5.7$ & 0.607 \\
\hline $\operatorname{Scr}(\mu \mathrm{mol} / \mathrm{l})$ & $80.6 \pm 44.8$ & $72.9 \pm 40.4$ & 0.492 \\
\hline $\mathrm{CrCl}(\mathrm{ml} / \mathrm{min})$ & $87.5 \pm 43.1$ & $100.0 \pm 53.9$ & 0.368 \\
\hline $\operatorname{ALT}(\mathrm{U} / \mathrm{l})$ & $36.5 \pm 26.7$ & $32.9 \pm 42.8$ & 0.732 \\
\hline $\operatorname{AST}(\mathrm{U} / \mathrm{l})$ & $45.4 \pm 42.8$ & $38.1 \pm 32.0$ & 0.451 \\
\hline WBC $\left(10^{9} / 1\right)$ & $12.3 \pm 5.0$ & $10.1 \pm 5.7$ & 0.141 \\
\hline Neutrophils (\% in WBCs) & $80.9 \pm 11.6$ & $79.6 \pm 11.0$ & 0.661 \\
\hline Lymphocytes (\% in WBCs) & $15.5 \pm 9.6$ & $16.0 \pm 9.4$ & 0.822 \\
\hline Albumin $(g / 1)$ & $29.8 \pm 6.6$ & $30.4 \pm 6.3$ & 0.687 \\
\hline Baseline body temperature $\left({ }^{\circ} \mathrm{C}\right)$ & $37.6 \pm 0.6$ & $38.2 \pm 1.0$ & 0.014 \\
\hline ICU patients & $12(60.0)$ & $28(62.2)$ & 0.865 \\
\hline Primary site of infection & & & 0.732 \\
\hline Respiratory tract & $16(80.0)$ & $34(75.6)$ & \\
\hline Bloodstream & $3(15.0)$ & $6(13.3)$ & \\
\hline Others & $1(5.0)$ & $5(11.1)$ & \\
\hline \multicolumn{4}{|l|}{ Initial anti-infective treatment } \\
\hline Carbapenems & $8(40.0)$ & $10(22.2)$ & 0.139 \\
\hline Cephalosporins & $3(15.0)$ & $11(24.4)$ & 0.393 \\
\hline \multicolumn{4}{|l|}{ Underlying disease } \\
\hline Cardiovascular & $14(70.0)$ & $21(46.7)$ & 0.082 \\
\hline Diabetes & $9(45.0)$ & $7(15.6)$ & 0.011 \\
\hline \multicolumn{4}{|l|}{ Vancomycin concentration (mg/l) } \\
\hline Trough & $18.2 \pm 7.0$ & $12.0 \pm 6.3$ & 0.001 \\
\hline Trough $\leq 15$ & $3(15.0)$ & $29(64.4)$ & $<0.001$ \\
\hline Trough $>15$ & $17(85.0)$ & $16(35.6)$ & \\
\hline Peak & $32.0 \pm 8.8$ & $26.6 \pm 8.3$ & 0.020 \\
\hline Total vancomycin dose (g) & $24.3 \pm 14.1$ & $17.7 \pm 10.6$ & 0.041 \\
\hline
\end{tabular}

Values are expressed as $\mathrm{n}(\%)$ or the mean \pm standard deviation. BMI, body mass index (normal range, 18.5-24.9 kg/m²); BUN, blood urea nitrogen (normal range, 3.2-7.1 mmol/l); Scr, serum creatinine (normal range, 44-133 $\mu \mathrm{mol} / \mathrm{l}$ ); $\mathrm{CrCl}$, creatinine clearance (normal range, 80-120 ml/min); ALT, alanine aminotransferase (normal range, 5-40 U/l); AST, aspartate transaminase (normal range, 8-40 U/l); WBC, white blood cell (normal range, 4-10x10 $/ 1$; ICU, intensive care unit. Neutrophils normal range, 50-70\%, lymphocytes normal range, $20-40 \%$ and albumin normal range $35-50 \mathrm{~g} / \mathrm{l}$.

association between nephrotoxicity and factor $4(\mathrm{OR}=2.388$; 95\% CI: 1.164-4.899; Table VI). Hence, higher initial trough and peak concentrations during vancomycin therapy bear a higher risk regarding the incidence of nephrotoxicity.

Prediction of the thresholds of vancomycin concentrations for efficacy. Fig. 2A presents ROC curves in which the vancomycin trough and peak concentrations were used as variables to predict the efficacy. The AUCs of the trough concentration and the peak concentration were 0.83 and 0.72 , respectively. The critical values of the trough concentration and peak concentration were $9.02 \mathrm{mg} / \mathrm{l}(95.3 \%$ sensitivity and $68.2 \%$ specificity) and $23.62 \mathrm{mg} / 1$ (83.7\% sensitivity and $59.1 \%$ specificity), respectively.
Table VI. Logistic regression analyses of independent risk factors for nephrotoxicity in the cohort $(n=65)$.

\begin{tabular}{lccc}
\hline Factor & $\begin{array}{c}\text { OR for } \\
\text { nephrotoxicity }\end{array}$ & $95 \% \mathrm{CI}$ & P-value \\
\hline 1 & 0.836 & $0.446-1.567$ & 0.577 \\
2 & 0.902 & $0.428-1.902$ & 0.787 \\
3 & 0.290 & $0.088-0.102$ & 0.051 \\
4 & 2.388 & $1.164-4.899$ & 0.018 \\
5 & 1.759 & $0.863-3.582$ & 0.120 \\
\hline
\end{tabular}

OR, odds ratio; CI, confidence interval. 

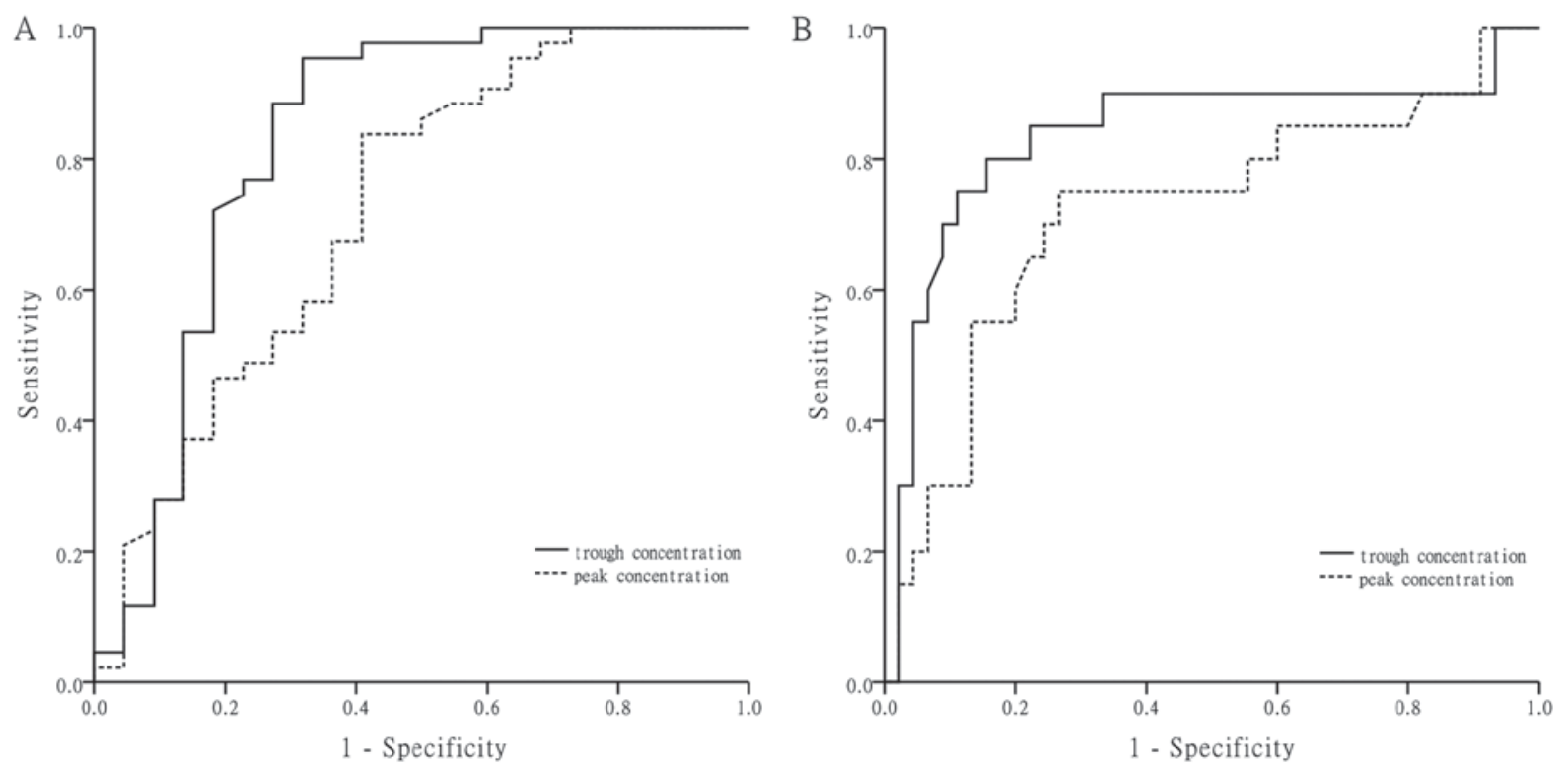

Figure 2. Receiver operating characteristic curves of vancomycin trough concentration and peak concentration. (A) Prediction of the critical value of vancomycin trough concentration and peak concentration for efficacy (cut-off value, 9.02 and $23.62 \mathrm{mg} / 1$, respectively); (B) prediction of the threshold of vancomycin trough concentration and peak concentration for nephrotoxicity (cut-off value, 16.08 and $30.42 \mathrm{mg} / 1$, respectively).

Prediction of the thresholds of vancomycin concentrations for nephrotoxicity. Fig. 2B presents the ROC curves for nephrotoxicity associated with the vancomycin trough and peak concentrations. The AUCs were 0.83 and 0.71 for the trough and peak concentration, respectively. The threshold vancomycin trough and peak concentrations for the development of nephrotoxicity were $16.08 \mathrm{mg} / \mathrm{l}$ (77.8\% sensitivity and $84.2 \%$ specificity) and $30.42 \mathrm{mg} / \mathrm{l}$ (72.2\% sensitivity and $76.3 \%$ specificity), respectively.

Based on the above results, a trough concentration between 9.02 and $16.08 \mathrm{mg} / \mathrm{l}$ and a peak concentration between 23.62 and $30.42 \mathrm{mg} / \mathrm{l}$ may be considered relatively safe, as these concentrations are not only effective but are also unlikely to induce nephrotoxicity.

\section{Discussion}

The present study investigated the predictive value of vancomycin serum concentrations regarding the drug's efficacy and nephrotoxicity. The results demonstrated that the differences in the trough concentration and peak concentration were statistically significant between the effective and ineffective groups, as well as between the nephrotoxicity and the non-nephrotoxicity groups. Furthermore, the critical values for the vancomycin serum concentration to achieve acceptable rates of efficacy and nephrotoxicity were identified.

Vancomycin was developed and approved in the 1950s for the treatment of infections with Gram-positive bacteria (16). Regarding the pharmacokinetics, $>90 \%$ of vancomycin is eliminated by the kidneys, and only $5-8.5 \%$ of vancomycin may be metabolized through hepatic conjugation (17). Renal elimination of vancomycin mostly occurs through glomerular filtration and to a certain extent through active tubular secretion (18). Dieterich et al (19) reported that vancomycin accumulates in proximal tubular cells, leading to cell necrosis as a mechanism of nephrotoxicity. Nishino et al (20) and
Oktem et al (21) suggested that oxidative stress and mitochondrial damage may contribute to vancomycin-associated renal injury. In addition to tubulointerstitial nephritis, severe vancomycin-induced nephrotoxicity may histologically manifest as granulomas in certain cases (22). The incidence of nephrotoxicity exhibits a wide variation and ranges from 5 to $>35 \%$ among various studies $(23,24)$. Therefore, the IDSA recommends that TDM is necessary to increase the rate of clinical efficacy and reduce the rate of nephrotoxicity during vancomycin therapy (3).

The bactericidal activity of vancomycin is thought to be time-dependent; therefore, it does not appear necessary to monitor peak concentrations. Suzuki et al (25) reported that it is not necessary to use peak concentrations of vancomycin in TDM, as the trough concentration/MIC and trough concentration ratio is sufficient to predict the efficacy and safety of vancomycin. However, there is support for a degree of concentration-dependent mortality associated with vancomycin (2). Iwamoto et al (26) indicated that monitoring of the peak concentration is essential for achieving an optimum clinical efficacy during vancomycin therapy, and a peak concentration of $>25 \mathrm{mg} / \mathrm{ml}$ may be more effective than peak concentrations $\leq 25 \mathrm{mg} / \mathrm{ml}$. In order to further elucidate the matter, the present study combined peak and trough concentrations during TDM to evaluate the efficacy and nephrotoxicity of vancomycin.

At present, the target vancomycin trough concentration for the optimum efficacy remains controversial. Chen et al (12) identified that the cut-off values of the first trough concentration were $7.9 \mathrm{mg} / \mathrm{l}$ for clinical efficacy and $21.1 \mathrm{mg} / \mathrm{l}$ for nephrotoxicity in Chinese patients. However, the IDSA provides recommendations that vancomycin trough concentrations should be maintained between 10 and $20 \mathrm{mg} / \mathrm{l}$ in order to avoid resistance and nephrotoxicity (3). In the present study, 80 samples from 65 patients were analyzed and only $58.0 \%$ were within the aforementioned range. The critical values for the trough concentration and peak concentration regarding 
efficacy were 9.02 and $23.62 \mathrm{mg} / \mathrm{l}$, respectively. The results demonstrate that the trough concentration together with the peak concentration provides a better assessment of the clinical efficacy of vancomycin than a trough concentration alone. In addition, it was identified that the BMI and albumin levels of the patients were associated with efficacy. Patients with poor nutrition may have serious infections, and normal doses of vancomycin may therefore not be effective.

It is well known that vancomycin has a significant nephrotoxicity; however, it remains elusive to what extent the vancomycin serum concentration is associated with nephrotoxicity. It has been demonstrated that an initial trough concentration of vancomycin of $\geq 15 \mathrm{mg} / \mathrm{l}$ and a duration of therapy of $\geq 14$ days are independent risk factors associated with higher rates of nephrotoxicity (27-29). A retrospective study including 1,269 cases reported that trough concentrations of $>12.1 \mathrm{mg} / \mathrm{l}$ were a major risk factor for vancomycin-induced nephrotoxicity (30). In the present study, the threshold vancomycin trough and peak concentrations for nephrotoxicity were determined to be 16.08 and $30.42 \mathrm{mg} / \mathrm{l}$, respectively. This result is slightly higher, but similar with that of previous studies, in terms of vancomycin trough concentrations increasing the efficacy and increasing the risk of nephrotoxicity.

Of note, the present study has a number of limitations. First, it was a single-center retrospective study with a small sample size. Furthermore, the patients treated with vancomycin generally had a variety of underlying conditions, but there was no homogeneity. In addition, the possibility of data observation bias cannot be excluded. In the future, studies using a larger sample and with more detailed stratification are required in order to identify the associations between serum vancomycin concentrations, efficacy and nephrotoxicity.

In conclusion, the present study provides evidence that vancomycin trough and peak concentrations are associated with the efficacy and incidence of nephrotoxicity of patients receiving vancomycin therapy. A trough concentration between 9.02 and $16.08 \mathrm{mg} / \mathrm{l}$ is relatively safe, and the relatively safe range for the peak concentration was from 23.62-30.42 mg/l. These results may provide useful information to guide the development of individualized vancomycin therapy.

\section{Acknowledgements}

The authors would like to thank Mr Zhang and Mr Tan at the Department of Respiratory Medicine of Shanghai 10th People's Hospital (Shanghai, China). The authors would also like to recognize and Mr Yuan at the Department of Laboratory Medicine of Shanghai 10th People's Hospital (Shanghai, China) who participated in the data collection for their cooperation and support. The authors are also grateful to Mr Liang at the Institute of Antibiotics of Huashan Hospital, Fudan University (Shanghai, China) for providing technical support.

\section{Funding}

This work was supported by the National Natural Science Foundation of China (grant no. 81472180). The funders had no role in the study design, data collection and analysis, decision to publish or preparation of the manuscript.

\section{Availability of data and materials}

The datasets used and/or analyzed during the present study are available from the corresponding author on reasonable request.

\section{Authors' contributions}

CHW and XLS conceived and designed the study, and critically revised the manuscript. LPW analyzed the data, interpreted the results and wrote the first draft of the manuscript. QY collected the clinical and laboratory data. MT and SSX were responsible for analysis of data and interpretation of results, as well as critical revision of the manuscript for important intellectual content. MT and SSX also approved the publication of the final manuscript. JFW was involved in detecting the vancomycin trough and peak concentrations. All authors read and approved the final manuscript.

\section{Ethics approval and consent to participate}

The study was approved by the Institutional Ethics Committee of the Shanghai Tenth People's Hospital of Tongji University.

\section{Patient consent for publication}

All the enrolled subjects gave informed consent for the present study.

\section{Competing interests}

The authors have declare that they have no competing interests.

\section{References}

1. National Nosocomial Infections Surveillance System: National Nosocomial Infections Surveillance (NNIS) System report, data summary from January 1992 through June 2004, issued October 2004. Am J Infect Control 32: 470-485, 2004.

2. Haque NZ, Zuniga LC, Peyrani P, Reyes K, Lamerato L, Moore CL, Patel S, Allen M, Peterson E, Wiemken T, et al: Relationship of vancomycin minimum inhibitory concentration to mortality in patients with methicillin-resistant Staphylococcus aureus hospital-acquired, ventilator-associated, or health-care-associated pneumonia. Chest 138: 1356-1362, 2010.

3. Martin JH, Norris R, Barras M, Roberts J, Morris R, Doogue M and Jones RD G: Therapeutic monitoring of vancomycinin adult patients: A consensus review of the American society of health-system pharmacists, the infectious diseases society of America, and the society of infectious diseases pharmacists. Clin Biochem Rev 31: 21-24, 2010.

4. Moellering RC Jr: Vancomycin: A 50-year reassessment. Clin Infect Dis 42 (Suppl 1): S3-S4, 2006.

5. Stevens DL: The role of vancomycin in the treatment paradigm. Clin Infect Dis 42 (Suppl 1): S51-S57, 2006.

6. Shah-Khan F, Scheetz MH and Ghossein C: Biopsy-proven acute tubular necrosis due to vancomycin toxicity. Int J Nephrol 2011: 436856, 2001.

7. Htike NL, Santoro J, Gilbert B, Elfenbein IB and Teehan G: Biopsy-proven vancomycin-associated interstitial nephritis and acute tubular necrosis. Clin Exp Nephrol 16: 320-324, 2012.

8. Matsumoto K, Takesue Y, Ohmagari N, Mochizuki T, Mikamo H, Seki M, Takakura S, Tokimatsu I, Takahashi Y, Kasahara K, et al: Practice guidelines for therapeutic drug monitoring of vancomycin: A consensus review of the Japanese society of chemotherapy and the Japanese society of therapeutic drug monitoring. J Infect Chemother 19: 365-380, 2013. 
9. Rybak M, Lomaestro B, Rotschafer JC, Moellering R Jr, Craig W, Billeter M, Dalovisio JR and Levine DP: Therapeutic monitoring of vancomycin in adult patients: A consensus review of the American society of health-system pharmacists, the infectious diseases society of America, and the society of infectious diseases pharmacists. Am J Health Syst Pharm 66: 82-98, 2009

10. Sakoulas G, Moise-Broder PA, Schentag J, Forrest A, Moellering RC Jr and Eliopoulos GM: Relationship of MIC and bactericidal activity to efficacy of vancomycin for treatment of methicillin-resistant Staphylococcus aureus bacteremia. J Clin Microbiol 42: 2398-2402, 2004.

11. Xin HW, Tong HY, Dong QR, Li Q, Wu XC, Yu AR, Xiong L and Li WL: Monitoring of blood concentration and individualized administration of vancomycin and norvancomycin in 207 cases. Chin J Pharmacoepidemiol 21: 166-169, 2012 (In Chinese).

12. Chen CY, Zhu SY, Zhou KT and Zhao YY, Xu P: Retrospective analysis of nephrotoxicity and efficacy of vancomycin trough concentrations in patients with severe pneumonia. Chin J Mod Appl Pharm 33: 1188-1194, 2016 (In Chinese).

13. Cockcroft DW and Gault MH: Prediction of creatinine clearance from serum creatinine. Nephron 16: 31-41, 1976.

14. Galbraith JI, Moustaki I, Bartholomew DJ and Steele F: The analysis and interpretation of multivariate data for social scientists. Chapman and Hall/CRC 56: 280, 2002.

15. Jolliffe IT: Principal Component Analysis. Springer, New York, NY, 1986.

16. Levine DP: Vancomycin: A history. Clin Infect Dis 42 (Suppl 1): S5-S12, 2006.

17. Matzke GR, Zhanel GG and Guay DR: Clinical pharmacokinetics of vancomycin. Clin Pharmacokinet 11: 257-282, 1986.

18. Nakamura T, Takano $M$, Yasuhara $M$ and Inui $K$ : In-vivo clearance study of vancomycin in rats. J Pharm Pharmacol 48: 1197-1200, 1996.

19. Dieterich C, Puey A, Lin S, Swezey R, Furimsky A, Fairchild D, Mirsalis JC and Ng HH: Gene expression analysis reveals new possible mechanisms of vancomycin-induced nephrotoxicity and identifies gene markers candidates. Toxicol Sci 107: 258-269, 2009.

20. Nishino Y, Takemura S, Minamiyama Y, Hirohashi K, Ogino $\mathrm{T}$, Inoue $\mathrm{M}$, Okada $\mathrm{S}$ and Kinoshita $\mathrm{H}$ : Targeting superoxide dismutase to renal proximal tubule cells attenuates vancomycin-induced nephrotoxicity in rats. Free Radic Res 37: $373-379,2003$
21. Oktem F, Arslan MK, Ozguner F, Candir O, Yilmaz HR, Ciris $\mathrm{M}$ and Uz E: In vivo evidences suggesting the role of oxidative stress in pathogenesis of vancomycin-induced nephrotoxicity: Protection by erdosteine. Toxicology 215: 227-233, 2005.

22. Hong S, Valderrama E, Mattana J, Shah HH, Wagner JD, Esposito M and Singhal PC: Vancomycin-induced acute granulomatous interstitial nephritis: Therapeutic options. Am J Med Sci 334: 296-300, 2007.

23. Lodise TP, Patel N,Lomaestro BM, Rodvold KA and Drusano GL: Relationship between initial vancomycin concentration-time profile and nephrotoxicity among hospitalized patients. Clin Infect Dis 49: 507-514, 2009.

24. Wong-Beringer A, Joo J, Tse E and Beringer P: Vancomycin-associated nephrotoxicity: A critical appraisal of risk with high-dose therapy. Int J Antimicrob Agents 37: 95-101, 2011.

25. Suzuki Y, Kawasaki K, Sato Y, Tokimatsu I, Itoh H, Hiramatsu K, Takeyama M and Kadota J: Is peak concentration needed in therapeutic drug monitoring of vancomycin? A pharmacokinetic-pharmacodynamic analysis in patients with methicillin-resistant staphylococcus aureus pneumonia. Chemotherapy 58: 308-312, 2012.

26. Iwamoto T, Kagawa Y and Kojima M: Clinical efficacy of therapeutic drug monitoring in patients receiving vancomycin. Biol Pharm Bull 26: 876-879, 2003.

27. Jeffres MN, Isakow W, Doherty JA, Micek ST and Kollef MH: A retrospective analysis of possible renal toxicity associated with vancomycin in patients with health care-associated methicillin-resistant Staphylococcus aureus pneumonia. Clin Ther 29: 1107-1115, 2007.

28. Bosso JA, Nappi J, Rudisill C, Wellein M, Bookstaver PB, Swindler J and Mauldin PD. Relationship between vancomycin trough concentrations and nephrotoxicity: A prospective multicenter trial. Antimicrob Agents Chemother 55: 5475-5479, 2011.

29. Liu Y, Yin Y, Liu XZ, Yao HJ, Li LX, Chen JH, Chen T, Lu XT, $\mathrm{Bu}$ SH and Zhang J: Retrospective analysis of vancomycin nephrotoxicity in elderly chinese patients. Pharmacology 95: 279-284, 2015.

30. Han HK, An H, Shin KH, Shin D, Lee SH, Kim JH, Cho SH, Kang HR, Jang IJ, Yu KS and Lim KS: Trough concentration over $12.1 \mathrm{mg} / \mathrm{l}$ is a major risk factor of vancomycin-related nephrotoxicity in patients with therapeutic drug monitoring. Ther Drug Monit 36: 606-611, 2014 